\title{
Three Dimensional Fluid Simulation and Experimental Verification of Hydrostatic Guideway for Large Rotary Worktable
}

\author{
Xue Jing Yu ${ }^{1}$, *, Zhao Ming ${ }^{1}$, Song Yang ${ }^{1}$, Wang Shao $\mathbf{L i}^{2}$ \\ ${ }^{1}$ Wuhan Heavy Duty Machine Tool Group Corporation, Wuhan, China \\ ${ }^{2}$ Mechanics Institute, Hunan Institute of Engineering, Xiangtan, China

\section{Email address:} \\ xuejingyu2504@126.com (Xue Jing Yu),wzhaom@126.com (Zhao Ming),wzsongyang@126.com (Song Yang), \\ 15130401@qq.com (Wang Shao Li) \\ ${ }^{*}$ Corresponding author
}

\section{To cite this article:}

Xue Jing Yu, Zhao Ming, Song Yang, Wang Shao Li. Three Dimensional Fluid Simulation and Experimental Verification of Hydrostatic Guideway for Large Rotary Worktable. Science Discovery. Vol. 6, No. 2, 2018, pp. 93-101. doi: 10.11648/j.sd.20180602.13

Received: April 20, 2018; Accepted: May 18, 2018; Published: June 22, 2018

\begin{abstract}
Relying on the major national science and technology special project, this study used the table 12 fan-shaped oil cavity hydrostatic guideway of CKS5235 CNC vertical lathe worktable, applied FLUENT processing software GAMBIT modeling and mesh generation the flow static pressure guide film, setting boundary conditions. Used simulation software FLUENT numerical analysis the dynamic process of hydrostatic slideway oil film flow field with temperature field and the basic bearing capacity, the effectiveness of three dimensional fluid simulation method for hydrostatic guideway in engineering design is verified by experiments. According to the results of three-dimensional fluid simulation, the hydrostatic guide structure of CKS5235 CNC vertical lathe worktable has been improved, achieve the speed performance improvement of the table, complete the major special technical research and acceptance.
\end{abstract}

Keywords: Rotary Table, Hydrostatic Guideway, High-Speed, Heavy Duty, Engineering Experiment

\section{大型回转工作台静压导轨三维流体仿真及实验验证}

薛敬宇 ${ }^{1 *}$, 赵明 ${ }^{1}$, 宋旸 ${ }^{1}$, 王少力 ${ }^{2}$

武汉重型机床集团有限公司, 武汉市, 中国

${ }^{2}$ 机械学院, 湖南工程学院, 湘潭市, 中国

\section{邮箱}

xuejingyu2504@126.com（薛敬宇）, wzhaom@126.com（赵明）, wzsongyang@126.com（宋旸）, 15130401@qq.com（王少力）

摘要：依托国家科技重大专项, 以CKS5235数控立式车床工作台12点扇形油腔静压导轨为研究对象, 运用FLUENT的 前处理软件GAMBIT对静压导轨油膜流场进行建模及网格划分, 设定边界条件。采用流体分析软件FLUENT数值模拟 了静压导轨油膜流场和温度场的动态变化过程及静压导轨的承载特性, 通过实验验证了静压导轨三维流体仿真方法在 工程设计中的有效性。根据三维流体仿真计算结果改进了CKS5235数控立式车床工作台静压导轨结构, 实现工作台转 速性能提升, 完成重大专项技术研究和验收。

关键词: 回转工作台, 静压导轨, 高速, 重载, 工程实验 


\section{1. 引言}

大型高速高精度回转工作台是高档数控机床的核心 部件, 广泛应用于航空、船舶、水电、核电、风电等行业 的高精度大型核心零件的加工。液体静压导轨以其精度高、 承载大、自适应能力强的特点, 被广泛用做大型高速高精 度回转工作台的支承导轨。

我国对大型高速高精度回转工作台的研究起步较晚, 产品性能与国外产品有较大差距, 其转速参数仅为国外同 规格产品的 $1 / 2$ 左右, 此类大型高速高精度回转工作台装 备主要依赖进口。因此提高国产大型高速高精度回转工作 台设计和制造水平, 对提升我国高档重型数控装备制造的 技术水平和国际竞争力具有重大意义。

近些年国内外学者对静压导轨技术进行了深入的研 究, 刘赵汬、金秋颖、张成印等以大尺寸液体静压支承转 台为研究对象, 模拟计算中引入温-粘及压-粘关系式, 计 算结果表明引入了温-粘及压-粘关系式的计算模型更符合 实际工况 [1]。邵俊鹏、张艳芹等针对重型静压轴承油 腔结构优化问题, 探讨了在转速、腔深及有效承载面积相 同时两种腔形的温度分布规律, 优化了油腔结构 [2][6]。 王少力、熊万里等针对重型液体静压转台的设计, 推导了 计入离心力因素的恒流供油扇形静压推力轴承承载力解 析计算公式 [3]。何发诚、桂林在恒流静压轴承系统中 引入调节静压轴承静压油流量的装置, 很好地解决了 DL250数控重型卧式镗车床静压轴承重载高精度的难题 [4]。邵俊鹏、张艳芹、李鹏程对重型立式数控车床工 作台静压导轨进行研究, 对比了不同形状油腔的流速分布 及压力分布, 揭示了导轨内部流动规律 [5]。张艳芹、 邵俊鹏、倪世钱对大尺寸静压推力轴承进行了仿真分析, 得到了静压轴承的压力及温度分布图 [7]。赵明、黄正 东、王书亭等针对预载恒流静压导轨的重型数控立车的工 作台由于承载大, 结构变形对油膜厚度的影响, 提出该计 算问题为静压-结构耦合问题, 通常采用迭代方法求解的 思想 [8］。王巧玲运用流体-固体耦合分析研究了温度和 压力分布对静压导轨静刚度的影响以及动态性能的影响 [9]。杨甫,余光怀,张力等. 以精密重载回转工作台恒流 闭式静压导轨为研究对象,结合静压系统典型故障分析, 优 化设计了静压导轨的结构参数、油膜厚度、油膜刚度,提 高了静压导轨的可靠性及运动精度 [10]。

由于工程用大型高速高精度回转工作台价格昂贵, 所 以, 结合大型高速高精度回转工作台实验的静压导轨特性 研究并不多。本课题来源于科技重大专项《超重型数控单 柱移动立式铣车床》 (No.2011ZX04002-091)的研究任务, 以专项的CKS5235数控立式车床工作台实验台为研究对 象, 展开大型回转工作台静压导轨三维流体仿真及实验验 证研究。通过实验验证了静压导轨三维流体仿真方法在工 程设计中的有效性。根据三维流体仿真结果改进了 CKS5235数控立式车床工作台静压导轨结构, 实现工作台 转速性能提升, 完成重大专项技术研究和验收。

\section{2. 离心力作用下的扇形油腔平面油垫设计理论}

CKS5235数控立式车床工作台 12 点扇形油腔静压导 轨单个扇形油垫示意图如图1所示。润滑油经各中心进油 孔流入扇形油腔后, 经四周封油边缝隙流出。图中, 阴影 部分表示单个扇形油垫的有效承载面积。 $Q_{1} 、 Q_{2} 、 Q_{3}$ 、 $Q_{4}$ 分别表示油垫各封油边的润滑油流量。 $R_{1} 、 R_{2} 、 R_{3}$ 、 $R_{4}$ 表示油垫及油腔各圆弧边对应的半径。 $R_{e 1} 、 R_{e 2}$ 表示 油垫的有效半径。 $\varphi_{1}$ 为扇形油垫对应的圆心角, $\varphi_{2}$ 为有 效承载面积区域对应的圆心角, $\varphi_{3}$ 为油腔对应的圆心角。 $b$ 为径向封油边宽度。

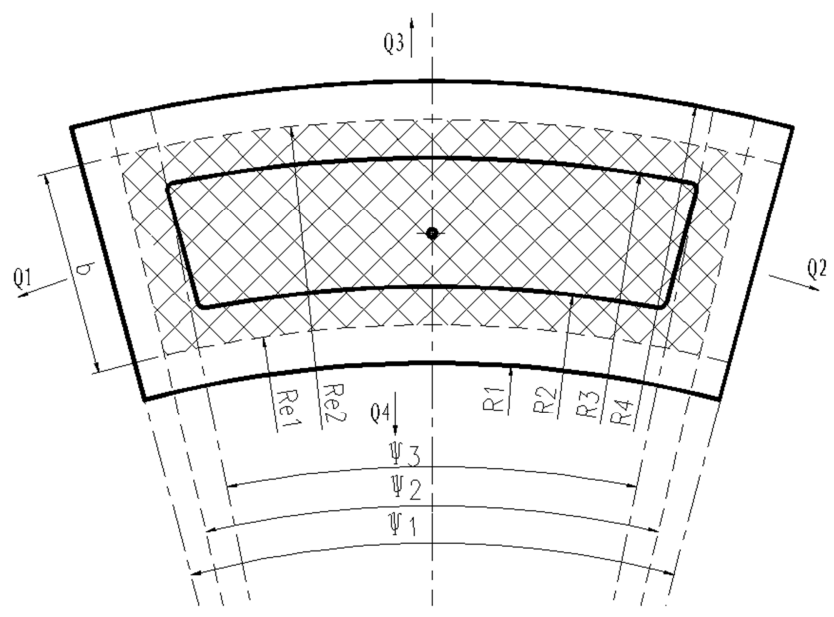

图1 扇形油垫示意图。

扇形油垫的有效夹角为

$$
\phi_{e}=\varphi_{2}=\left(\varphi_{1}+\varphi_{3}\right) / 2
$$

有效承载面积外圆半径为

$$
R_{e 1}=R_{4} \sqrt{\frac{1-\left(R_{3} / R_{4}\right)^{2}}{2 \ln \left(R_{4} / R_{3}\right)}}
$$

有效承载面积内圆半径为

$$
R_{e 2}=R_{2} \sqrt{\frac{1-\left(R_{1} / R_{2}\right)^{2}}{2 \ln \left(R_{2} / R_{1}\right)}}
$$

有效承载区径向宽度为

$$
b=R_{e 1}-R_{e 2}
$$

当量半径为

$$
R_{e}=\left(R_{e 1}+R_{e 2}\right) / 2
$$

两直线封油边上游流量 $Q_{1}$ 及下游流量 $Q_{2}$ 分别为 


$$
\begin{gathered}
Q_{1}=\frac{\left(R_{e 1}-R_{e 2}\right) h^{3} \Delta p}{6 \mu\left(\varphi_{1}-\varphi_{3}\right) R_{e}}-\frac{1}{2} b h \omega R_{e} \\
Q_{2}=\frac{\left(R_{e 1}-R_{e 2}\right) h^{3} \Delta p}{6 \mu\left(\varphi_{1}-\varphi_{3}\right) R_{e}}+\frac{1}{2} b h \omega R_{e}
\end{gathered}
$$

考虑离心力作用, 周向外圆弧封油边流量 $Q_{3}$ 与内圆 弧封油边流量 $Q_{4}$ 分别为

$$
\begin{gathered}
Q_{3}=\frac{\phi_{e} h^{3}\left[\Delta p+0.15 \rho \omega^{2}\left(R_{4}^{2}-R_{3}^{2}\right)\right]}{12 \mu \ln \left(R_{4} / R_{3}\right)} \\
Q_{4}=\frac{\phi_{e} h^{3}\left[\Delta p-0.15 \rho \omega^{2}\left(R_{2}^{2}-R_{1}^{2}\right)\right]}{12 \mu \ln \left(R_{2} / R_{1}\right)}
\end{gathered}
$$

由公式1-4, 求得供油量为

$$
\begin{gathered}
Q_{0}=Q_{1}+Q_{2}+Q_{3}+Q_{4} \\
=\frac{b h^{3} \Delta p}{3 \mu\left(\varphi_{1}-\varphi_{3}\right) R_{e}}+\frac{\phi_{e} h^{3} \Delta p}{12 \mu} \times \frac{\ln \left(R_{4} R_{2} / R_{3} R_{1}\right)}{\ln \left(R_{4} / R_{3}\right) \ln \left(R_{2} / R_{1}\right)} \\
+\frac{0.15 \rho \omega^{2} \phi_{e} h^{3}}{6 \mu}\left(R_{e 1}^{2}-R_{e 2}^{2}\right) \\
=C_{1} \frac{h^{3} \Delta p}{\mu}+C_{2} \frac{h^{3} \Delta p}{\mu}+C_{3} \frac{\phi_{e} b R_{e} \omega^{2} h^{3}}{\mu} \\
C_{1}=\frac{b}{3\left(\varphi_{1}-\varphi_{3}\right) R_{e}} ; \\
C_{2}=\frac{\phi_{e} \ln \left(R_{4} R_{2} / R_{3} R_{1}\right)}{12 \ln \left(R_{4} / R_{3}\right) \ln \left(R_{2} / R_{1}\right)} ; \\
C_{3}=0.05 \rho
\end{gathered}
$$

式中,

对于恒流供油静压轴承, $Q_{0}$ 始终为一定值。当油膜 间隙一定时, 油腔内压强为

$$
\Delta p=\frac{\mu Q_{0}-C_{3} \phi_{e} b R_{e} \omega^{2} h^{3}}{\left(C_{1}+C_{2}\right) h^{3}}
$$

扇形油垫的有效承载面积为

$$
A_{e}=\frac{\phi_{e}}{2}\left[R_{4}{ }^{2} \frac{1-\left(R_{3} / R_{4}\right)^{2}}{2 \ln \left(R_{4} / R_{3}\right)}-R_{2}{ }^{2} \frac{1-\left(R_{1} / R_{2}\right)^{2}}{2 \ln \left(R_{2} / R_{1}\right)}\right]
$$

单个油垫的承载力为

$$
W=\frac{\mu Q_{0} A_{e}}{\left(C_{1}+C_{2}\right) h^{3}}-\frac{C_{3} \phi_{e} b R_{e} \omega^{2} A_{e}}{C_{1}+C_{2}}
$$

\section{CKS5235数控立式车床工作台的静压导轨结} 构

CKS5235数控立式车床工作台的静压导轨呈环形, 如 图2所示。环形静压导轨由 12 个扇形油垫等角度分布, 每 个扇形油垫的结构尺寸均相同。

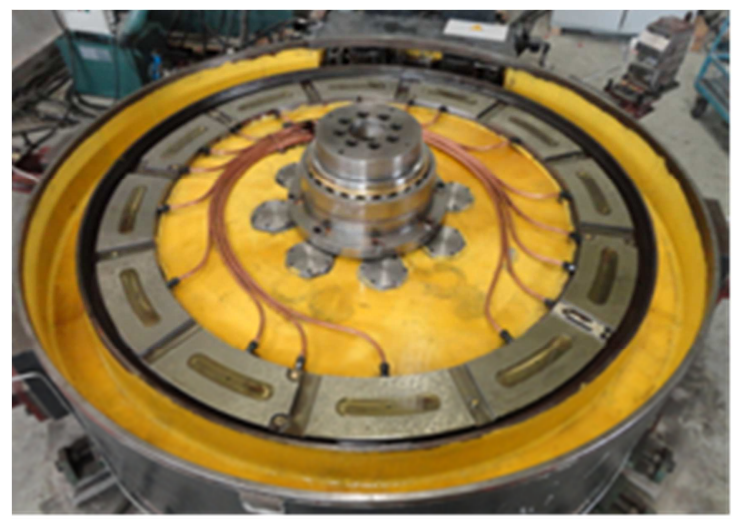

图2 CKS5235数控立式车床工作台静压导轨。

其单个扇形油垫的结构尺寸, 如图3所示。

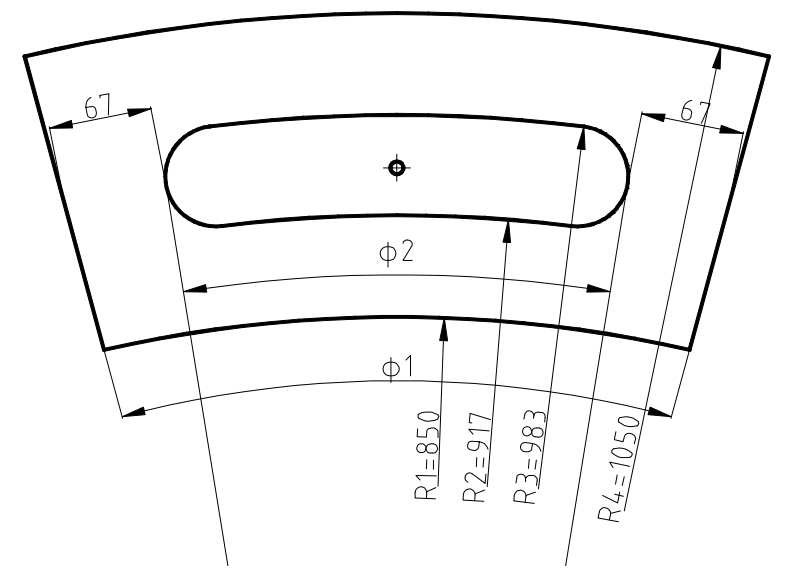

图3 CKS5235静压导轨单个扇形油垫尺寸结构图。

\section{CKS5235工作台扇形油腔静压导轨三维流体 仿真}

平行状态下转动的CKS5235机床工作台静压导轨, 12 个扇形油垫承载特性相同, 本节仅对单个扇形油垫进行建 模与仿真, 分析静压导轨的动、静态特性。

\section{1. 静压导轨的单个油垫内流体流速分布云图随转速的 变化过程}

CKS5235工作台静压导轨的单个油垫内流体流速分 布云图随转速的变化过程, 如图4所示。该图以进油温度 为 $30^{\circ} \mathrm{C}$, 油洜流量为 $16 \mathrm{~L} / \mathrm{min}$, 油膜间隙为 $0.12 \mathrm{~mm}$ 工况为 例, 取油膜高度为 $0.11 \mathrm{~mm}$ 平面上的速度矢量分布。 


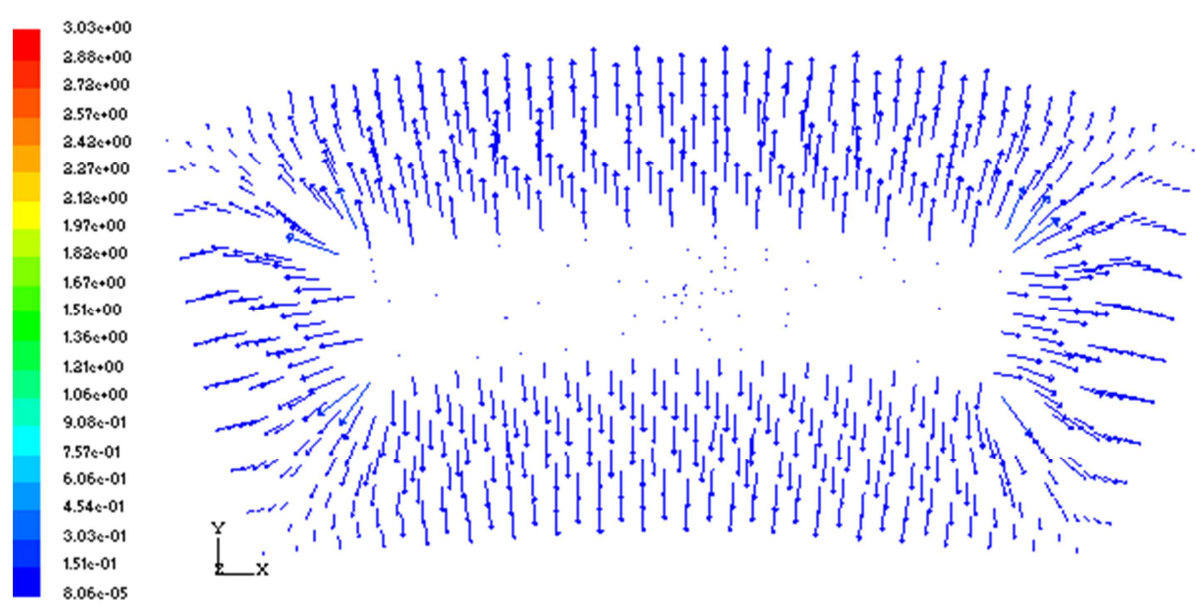

(a) 转速为 $0 \mathrm{rad} / \mathrm{s}$ 时, 油膜高度 $\mathrm{Z}=0.11 \mathrm{~mm}$ 平面上的速度矢量分布图

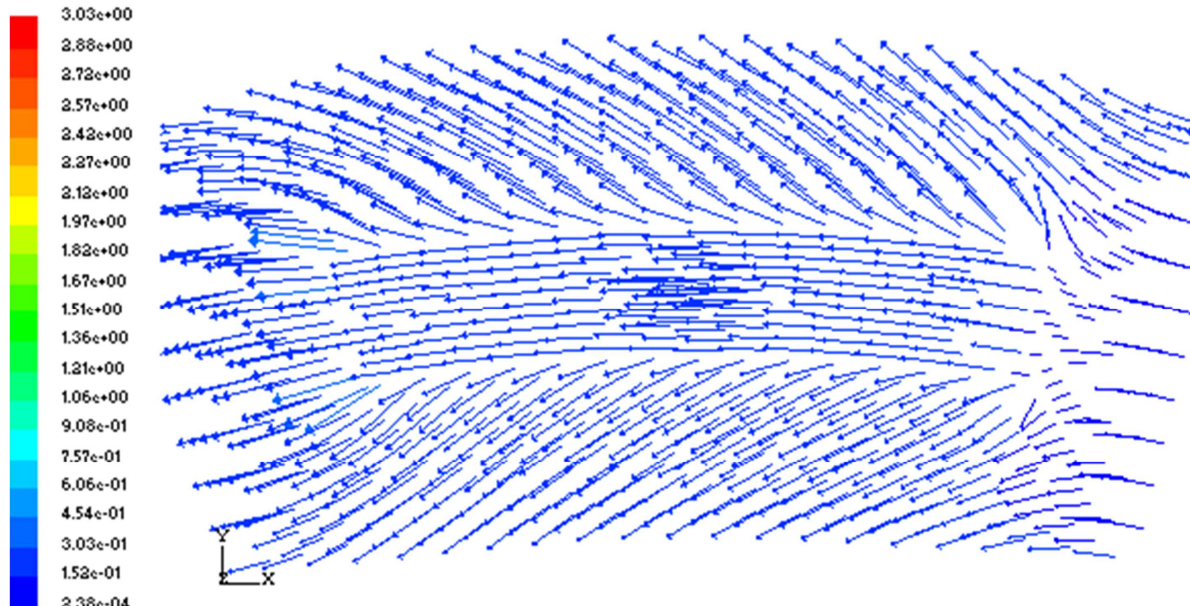

(b) 转速为 $0.2 \mathrm{rad} / \mathrm{s}$, 油膜高度 $\mathrm{Z}=0.11 \mathrm{~mm}$ 平面上的速度矢量分布图
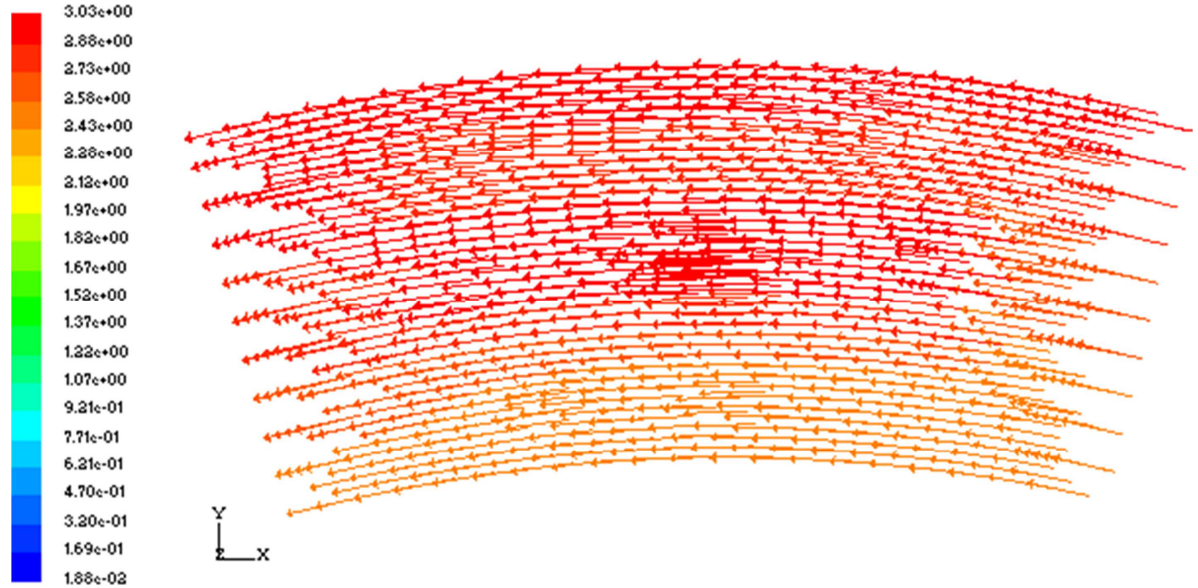

(c) 转速为 $3.14 \mathrm{rad} / \mathrm{s}$, 油膜高度 $\mathrm{Z}=0.11 \mathrm{~mm}$ 平面上的速度矢量分布图

图4 速度矢量分布随转速的变化过程.

图4（a）显示，转速为 $0 \mathrm{rad} / \mathrm{s}$ 时，速度矢量分布符合 平行平板间的缝隙流理论, 在导轨内外压差作用下, 液体 形成压差流, 由导轨进油孔向导轨边沿流出。图4（b）显 示, 转速为 $0.2 \mathrm{rad} / \mathrm{s}$ 时, 在导轨内外压差和导轨移动剪切作 用下, 液体形成压差-剪切流。对比转速为 $0 \mathrm{rad} / \mathrm{s}$ 时的速度 矢量分布, 转速为 $0.2 \mathrm{rad} / \mathrm{s}$ 时的速度矢量明显叠加了一个圆 周切向矢量, 导轨液体流动方向和速度发生改变, 在导轨
右端边沿处液体反向流动, 整个过程符合有相对运动的平 行平板间的缝隙流理论。图4（b) 和图4（c）对比显示, 转速由 $0.2 \mathrm{rad} / \mathrm{s}$ 提高到 $3.14 \mathrm{rad} / \mathrm{s}$ 时, 随着转速的提高, 导轨 移动剪切作用对液体流动速度和流动方向的影响更加明 显。 
4.2. 静压导轨的单个油垫内流体压强分布随转速的变化 过程

油膜厚度为 $0.12 \mathrm{~mm}$ 、进油温度为 $30^{\circ} \mathrm{C}$, 油原流量为

$16 \mathrm{~L} / \mathrm{min}$ 工况下, 考虑粘度随温度变化时, CKS5235型液
体静压转台的单个油垫内流体压强分布随转速的变化过 程, 如图5所示。
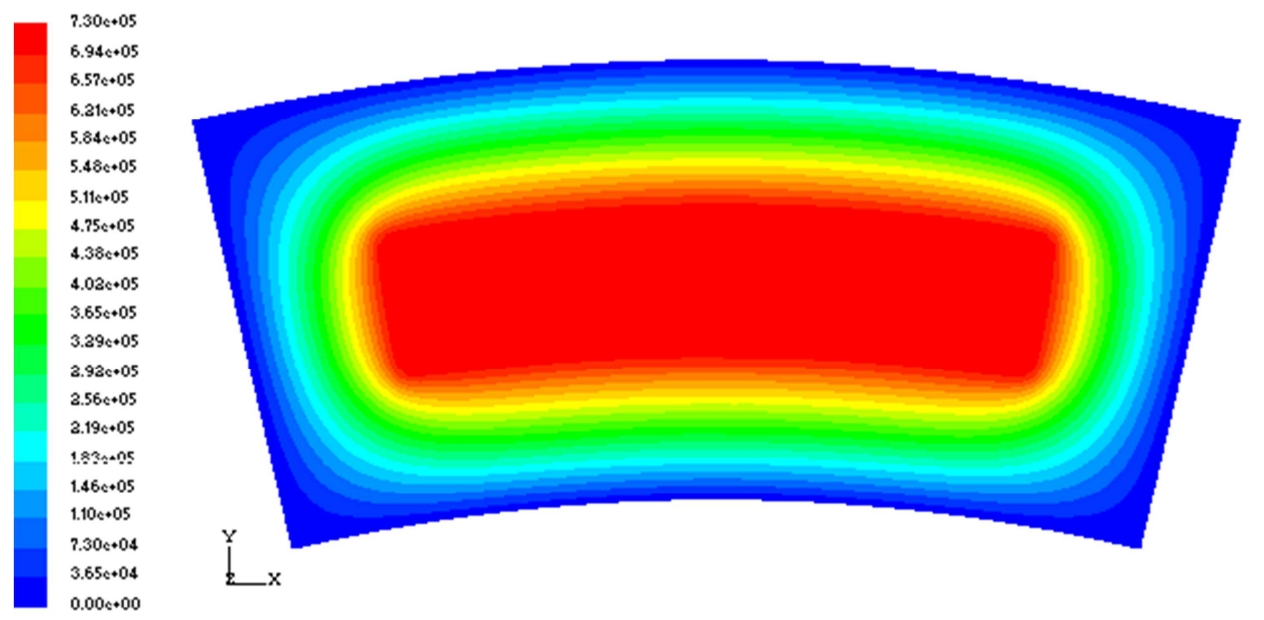

(a) 转速为 $0 r / m i n$ 时, 压强分布云图

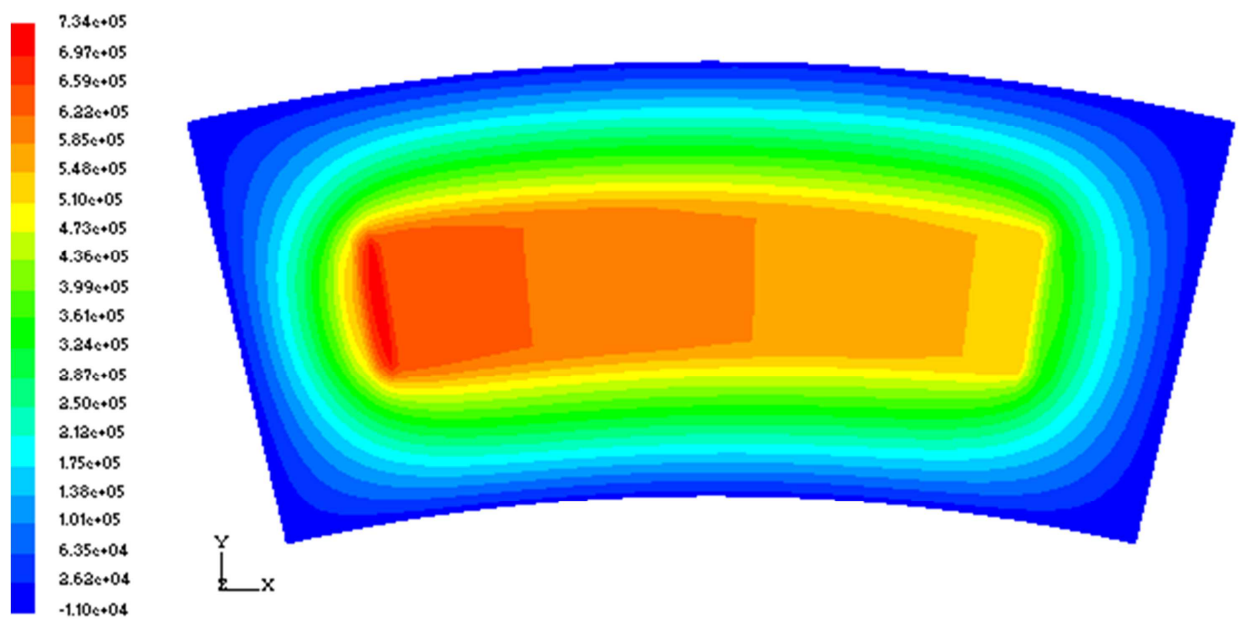

(b) 转速为 $50 \mathrm{r} / \mathrm{min}$ 时, 压强分布云图
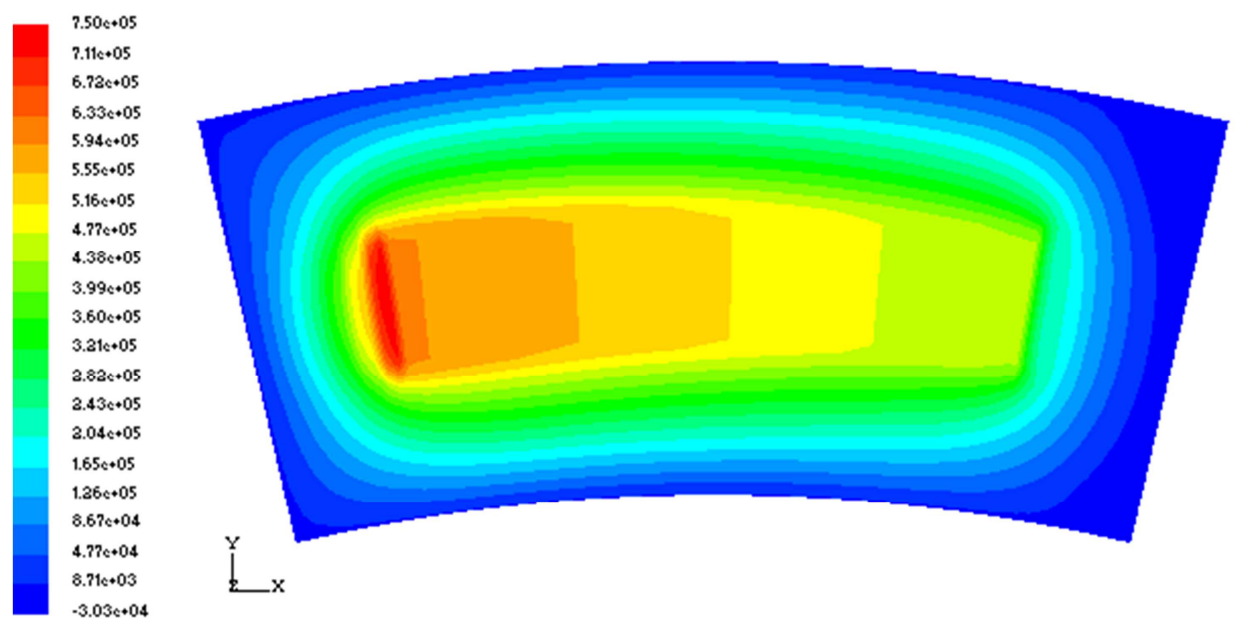

(c) 转速为 $80 \mathrm{r} / \mathrm{min}$ 时, 压强分布云图

图5 压强分布云图随转速变化过程. 
图5（a）显示, 转速为 $0 \mathrm{r} / \mathrm{min}$ 时, 压强分布云图符合 平行平板间的缝隙流理论, 导轨压强值由内向外递减。图 5（b) 和图 5 (c) 显示, 转速在 $50 \mathrm{r} / \mathrm{min}$ 和 $80 \mathrm{r} / \mathrm{min}$ 的工况 下, 导轨油腔内出现动压现象, 随着转速的增加, 动压现 象越明显。

\section{3. 静压导轨的单个油垫内流体温度分布随转速的变化 过程}

油膜厚度为 $0.12 \mathrm{~mm}$ 、进油温度为 $30^{\circ} \mathrm{C}$, 油泵流量为 $16 \mathrm{~L} / \mathrm{min}$ 工况下, CKS5235型液体静压转台的单个油垫内 油膜高度为 $0.11 \mathrm{~mm}$ 平面上的流体温度分布随转速的变化 过程, 如图6所示。
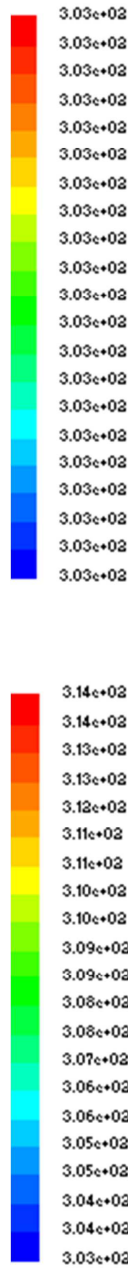

$.14 \mathrm{c}+03$

$3.13 \div+02$

$3.13 \mathrm{c}+02$

$3.11 \div+02$

$3.11,+03$

$3.10 \mathrm{~s}+03$

$.10 \mathrm{~s} \div 02$

$09 \mathrm{c}+02$

$.09 \mathrm{c}+02$

$08 \div 02$

$.07 \div 002$

$3.06 \div+02$

$.05 \div \div 02$

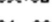

$.03 \times \cdot 08$

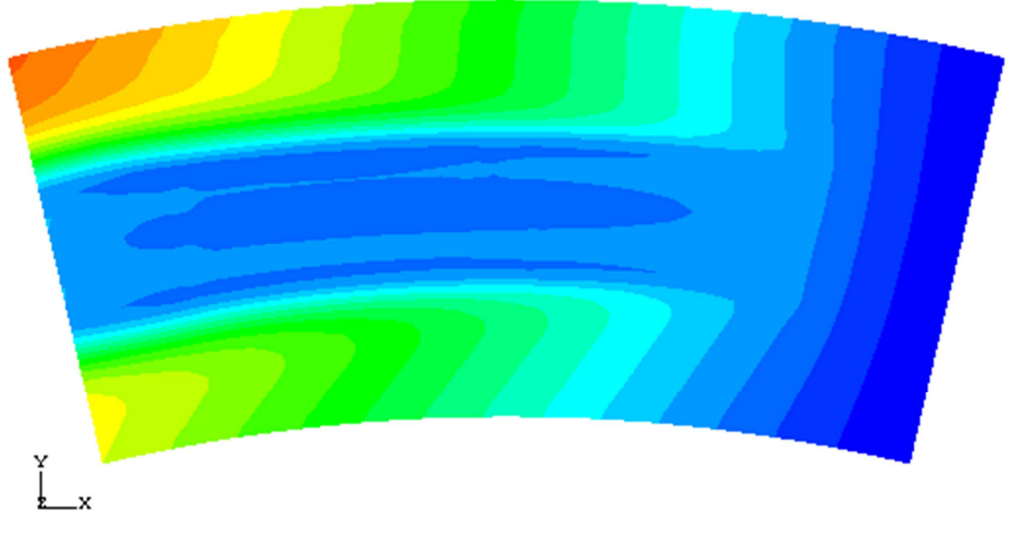

(b) 转速为 $50 \mathrm{r} / \mathrm{min}$ 时, 油膜高度 $\mathrm{Z}=0.11 \mathrm{~mm}$ 平面上的温度分布云图
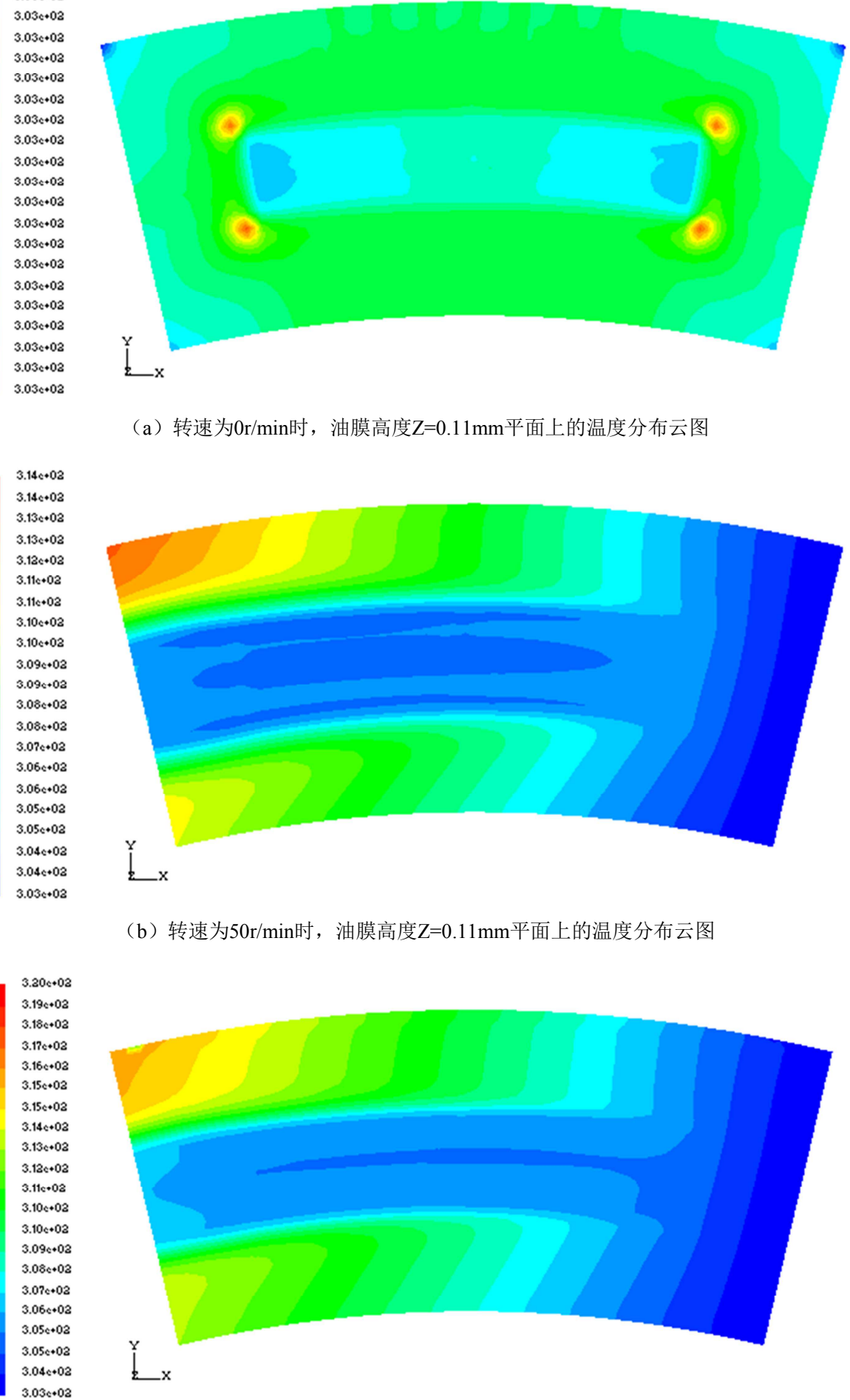

(a) 转速为 $0 \mathrm{r} / \mathrm{min}$ 时, 油膜高度 $\mathrm{Z}=0.11 \mathrm{~mm}$ 平面上的温度分布云图

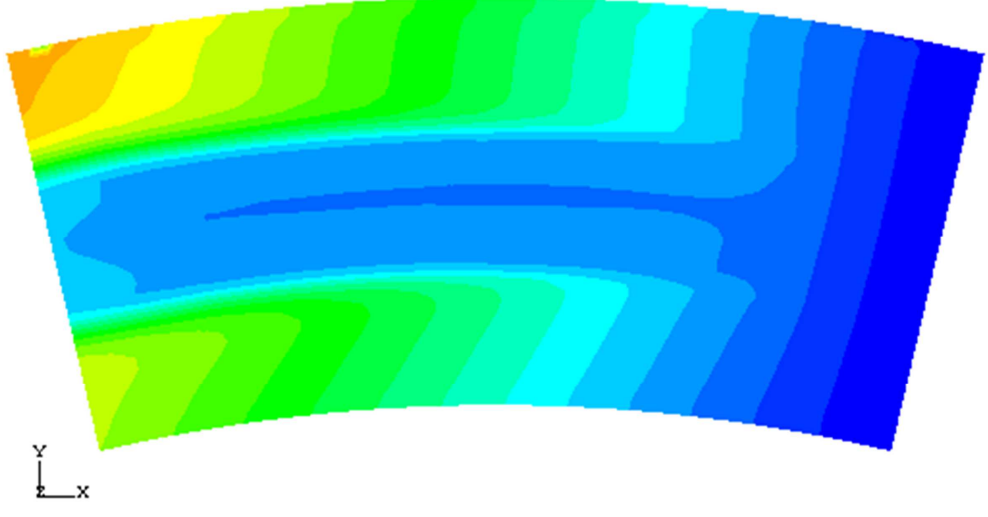

(c) 转速为 $80 \mathrm{r} / \mathrm{min}$ 时, 油膜高度 $\mathrm{Z}=0.11 \mathrm{~mm}$ 平面上的温度分布云图

图6 温度分布云图随转速的变化过程。 
图6（a）显示, 转速为 $0 \mathrm{r} / \mathrm{min}$, 转台静止时, 流体不 受摩擦剪切做功, 温度基本无变化。图6（b）显示, 转速 为 $50 \mathrm{r} / \mathrm{min}$ 时, 转动状态下, 流体由于受摩擦剪切做功, 产 生热量, 流体温度升高。图6（b) 和图6（c）对比显示, 转速由 $50 \mathrm{r} / \mathrm{min}$ 提高到 $80 \mathrm{r} / \mathrm{min}$ 时, 随着转速的提高, 随着 转速的增加, 流体受摩擦剪切做功越多, 产生热量越多, 流体温度越高。
为了研究CKS5235数控立式车床工作台静压导轨油 膜的速度场、压力场和温度场之间的关系。在CKS5235立 式数控车床液体静压实验转台进油温度为 $30^{\circ} \mathrm{C}$ 、油膜间隙 为 $0.12 \mathrm{~mm}$ 工况下, 分别对油原流量为 $16 \mathrm{~L} / \mathrm{min} 、 30 \mathrm{~L} / \mathrm{min}$, 转速为 $0 \mathrm{r} / \mathrm{min} 、 32 \mathrm{r} / \mathrm{min} 、 40 \mathrm{r} / \mathrm{min} 、 50 \mathrm{r} / \mathrm{min} 、 63 \mathrm{r} / \mathrm{min} 、 80 \mathrm{r} / \mathrm{min}$ 、 $100 \mathrm{r} / \mathrm{min}$ 、 $120 \mathrm{r} / \mathrm{min}$ 等情况下的单个油垫的润滑油流场进 行了三维仿真研究, 仿真计算结果见表 1 。

表 $130^{\circ} \mathrm{C}$, 油膜间隙为 $0.12 \mathrm{~mm}$, 不同油泉流量、不同转速情况下, 转台性能的仿真值计算结果。

\begin{tabular}{|c|c|c|c|c|c|c|}
\hline 流量 & $16 \mathrm{~L} / \mathrm{min}$ & & & 30L/min & & \\
\hline 转台转速(r/min) & 总承载能力(T) & 腔内最大压(Mpa) & 直线边油温(K) & 总承载能力(T) & 腔内最大压强(Mpa) & 直线边油温(K) \\
\hline 0 & 40.78 & 0.73 & 303 & 76.34 & 1.37 & 303 \\
\hline 32 & 35.47 & 0.73 & 306.50 & 67.68 & 1.29 & 305.56 \\
\hline 50 & 32.44 & 0.73 & 309.03 & 62.40 & 1.26 & 307.89 \\
\hline 63 & 30.43 & 0.74 & 310.66 & 58.80 & 1.24 & 309.57 \\
\hline 80 & 27.88 & 0.75 & 312.59 & 54.60 & 1.22 & 311.56 \\
\hline 100 & 24.86 & 0.76 & 314.63 & 50.04 & 1.21 & 313.67 \\
\hline 120 & 21.73 & 0.77 & 316.46 & 45.60 & 1.20 & 315.55 \\
\hline
\end{tabular}

表1数据表明, 静压导轨在润滑油流量、进油温度、 油膜间隙不变的情况下, 随着转台转速的提高, 摩擦剪切 做功增加, 油膜温度升高, 润滑油粘度降低, 油膜的承载 能力下降。

通过对仿真计算结果的分析, 可以得出以下结论:

CKS5235数控立式车床工作台, 工程上允许的最高转 速为 $63 \mathrm{r} / \mathrm{min}$ 。仿真结果表明, 理论上该转台转速有提升空 间, 可以提高到 $120 \mathrm{r} / \mathrm{min}$ (转速 $120 \mathrm{r} / \mathrm{min}$ 时, 油膜温度低 于工程许用最高油温 $318 \mathrm{~K}$ 为判定依据）。但转速 $120 \mathrm{r} / \mathrm{min}$ 时, 油膜温度总体较高, 从工程应用的角度考虑, 静压导 轨的结构可进一步优化, 以降低导轨油膜温度。

\section{CKS5235数控立式车床工作台实验研究}

\section{1. 静止状态下的实验}

调节供油量, 工作台不旋转工况下, 进行流量、压力、 油膜厚度数据记录。

静止状态下, 由于油膜没有发生剪切做功, 润滑油温 度不变, 粘度不变, 满足无粘温效应的理想假设条件。因 此, 静止状态下的实验可用于研究无粘温效应的理想假设 条件下, 油膜间隙与承载力及油腔压强的关系。

\section{2. 转动状态下的实验}

调节供油量, 使CKS5235数控立式车床工作台在某一 转速下持续工作, 当静压转台导轨温度恒定以后, 检测并 记录回转工作台转速、润滑油流量、油膜间隙和导轨温度 等实验数据。

转动状态下, 由于油膜发生剪切做功, 润滑油温度升 高。将实验测得的数据与考虑粘温效应的三维仿真结果进 行比较, 研究考虑粘温效应时, 液体静压回转工作台的转 速对润滑油温升的影响规律。

\section{6. 实验结果与仿真结果的对比分析}

\section{1. 静态浮升实验}

将实验测得的数据通过计算转化为实际的相关物理 量数值, 并将其与三维仿真值进行对比。现分析如下:

载荷一定时, 实验数据与三维仿真结果的对比, 在表 2 中列举了载荷为 21 吨, 流量分别为 $6.66 \mathrm{~L} / \mathrm{min} 、 7.07 \mathrm{~L} / \mathrm{min}$ 、 $8.63 \mathrm{~L} / \mathrm{min} 、 11.6 \mathrm{~L} / \mathrm{min}$ 时, 相关的实验数据和仿真计算结 果。

表 2 载荷为 21 吨, 进油温度为 $32.5^{\circ} \mathrm{C}$ 时, 实验数据与理论计算、仿真计算结果对比表。

\begin{tabular}{|c|c|c|c|c|c|c|c|}
\hline \multirow{2}{*}{ 编号 } & \multicolumn{3}{|l|}{ 实验数据 } & \multicolumn{4}{|c|}{ 三维仿真结果 } \\
\hline & 流量(L/min) & 位移(mm) & 压强(MPa) & 压强(MPa) & 压强误差 & 承载(T) & 承载误差 \\
\hline 1 & 6.66 & 0.097 & 0.358 & 0.365 & $2 \%$ & 19.89 & $5.5 \%$ \\
\hline 2 & 7.07 & 0.101 & 0.35 & 0.378 & $8 \%$ & 21.14 & $0.7 \%$ \\
\hline 3 & 8.63 & 0.107 & 0.355 & 0.356 & $0.2 \%$ & 19.89 & $5.5 \%$ \\
\hline 4 & 11.6 & 0.119 & 0.395 & 0.382 & $3 \%$ & 21.3 & $1.4 \%$ \\
\hline
\end{tabular}

通过对实验数据与仿真计算结果的比较分析, 可以得 出以下结论:

无粘温效应的理想条件下，如表2所示，三维仿真得 到的压强值与实验数据对比, 偏大 $8 \%$ 以内, 承载力相差 在 $5.5 \%$ 以内。表明, 无粘温效应的理想条件下, 三维仿真 计算结果可以作为工程计算的理论依据。

\section{2. 转动状态下, 温升实验}

\subsection{1. 静压导轨油腔结构改进前, 转动状态下, 温升实验}

表 3 为进油温度为 $24^{\circ} \mathrm{C}$ 时, 实验测得的静压转台导轨 板温度与考虑润滑油的粘温效应的仿真计算值对比。图7 为流量为 $7 \mathrm{~L} / \mathrm{min}$, 进油温度为 $24^{\circ} \mathrm{C}$ 时, 实验与仿真计算得 到的温升与转速的关系曲线图。 
表3 进油温度 $24^{\circ} \mathrm{C}$ 时, 实验测得导轨板温度与仿真计算值对比。

\begin{tabular}{|c|c|c|c|c|c|c|c|}
\hline \multirow{2}{*}{$\begin{array}{l}\text { 转速 } \\
\text { (r / m i n) }\end{array}$} & \multirow{2}{*}{$\begin{array}{l}\text { 流量 } \\
\text { (r / m i n) }\end{array}$} & \multirow{2}{*}{$\begin{array}{l}\text { 油膜间隙 } \\
(\mathrm{mm})\end{array}$} & \multicolumn{2}{|l|}{ 实验数据 } & \multicolumn{3}{|l|}{ 仿真数据 } \\
\hline & & & 导轨温度 $\left({ }^{\circ} \mathrm{C}\right)$ & 导轨温升 $\left({ }^{\circ} \mathrm{C}\right)$ & 导轨温升 $\left({ }^{\circ} \mathrm{C}\right)$ & 导轨温升误差 & 承载力 $(T)$ \\
\hline 60 & 5.32 & 0.085 & 42.6 & 18.6 & 17.2 & $8.5 \%$ & 26.3 \\
\hline 65 & 6.72 & 0.095 & 40.5 & 16.5 & 15.8 & $4.2 \%$ & 32.4 \\
\hline 70 & 6.89 & 0.094 & 42.6 & 18.6 & 16.6 & $11 \%$ & 32.2 \\
\hline 80 & 7.75 & 0.094 & 44.4 & 20.4 & 17.9 & $13 \%$ & 34.2 \\
\hline 90 & 7.80 & 0.095 & 46.8 & 22.8 & 19.2 & $16 \%$ & 32.4 \\
\hline
\end{tabular}

通过对比分析表3中实验数据和仿真计算结果及图7 中温升与转速的曲线图, 可以得出以下结论:

(1)在以上各不同转速和流量工况下, 仿真计算得到的 液体静压转台导轨温度与实测导轨温度误差分别为 $8.5 \%$ 、 $4.2 \% 、 11 \% 、 13 \%$ 和 $16 \%$ 。说明液体静压转台在转动状态 下, 考虑粘温效应的三维仿真计算得到的静压导轨温度与 工程实际相吻合, 证明对静压回转工作台的流体三维建模、
网格划分方法及边界条件设置是合理的, 利用Fluent软件 对静压导轨温升进行数值模拟计算方法是有效可行的。

(2)实验测得的静压导轨温升与转速的关系曲线图与 仿真计算得到的静压导轨温升与转速的关系曲线图趋势 一致。如图7所示, 液体静压转台导轨的温升随着转速的 增大而增大。

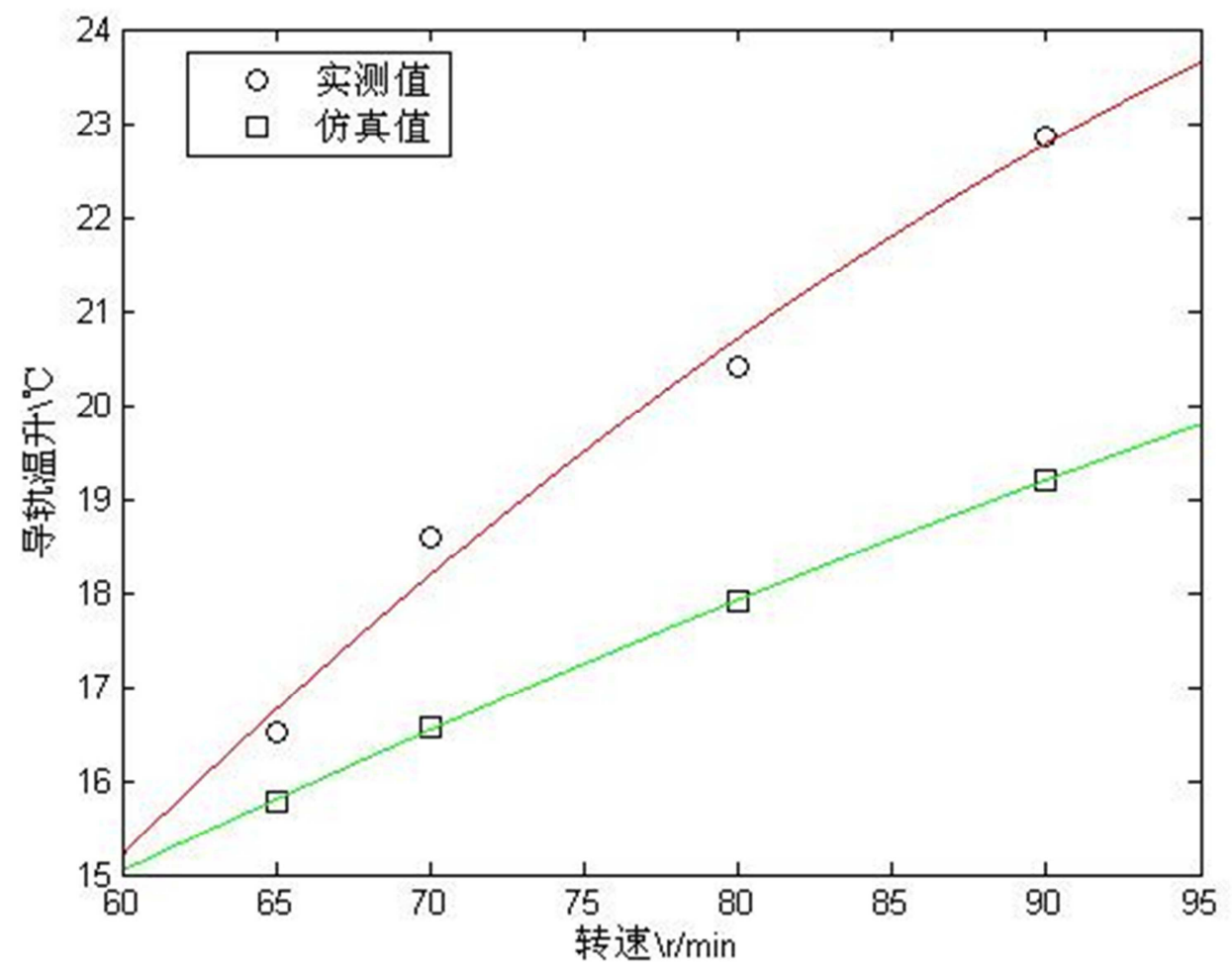

图7 流量为 $7 \mathrm{~L} / \mathrm{min}$, 进油温度为 $24^{\circ} \mathrm{C}$ 时, 温升与转速的关系。

\subsection{2. 静压导轨油腔结构改进后, 转动状态下, 温升实验}

表4 进油温度 $29^{\circ} \mathrm{C}$ 时, 实验测得导轨板温度。

\begin{tabular}{|c|c|c|c|c|}
\hline \multirow{2}{*}{$\begin{array}{l}\text { 转速 } \\
(\mathrm{r} / \mathrm{min})\end{array}$} & \multirow{2}{*}{$\begin{array}{l}\text { 流量 } \\
\text { (L/min) }\end{array}$} & \multirow{2}{*}{ 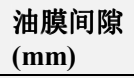 } & \multicolumn{2}{|l|}{ 实验数据 } \\
\hline & & & 温度 $\left({ }^{\circ} \mathrm{C}\right)$ & 温升 $\left({ }^{\circ} \mathrm{C}\right)$ \\
\hline 90 & 19.24 & 0.095 & 36.7 & 7.7 \\
\hline 100 & 22.92 & 0.095 & 37.3 & 8.3 \\
\hline 110 & 25.51 & 0.096 & 38.4 & 9.4 \\
\hline 120 & 28.94 & 0.094 & 39.1 & 10.1 \\
\hline
\end{tabular}

通过表4数据可以说明, 静压导轨油腔结构改进后, CKS5235数控立式车床工作台实现转速 $120 \mathrm{r} / \mathrm{min}$ 稳定运转, 导轨油膜温度为 $39.1^{\circ} \mathrm{C}$ 。

\section{7. 结论}

本文对CKS5235数控立式车床工作台静压导轨进行 三维流体仿真及实验验证研究, 得出以下主要结论:

(1) 采用三维流体仿真方法得到了不同工况下 CKS5235数控立式车床工作台静压导轨油膜的速度场、压 力场和温度场。通过对比实验数据, 验证了三维流体仿真 结果正确, 三维仿真计算方法贴近工程实际, 对该类立式 车床工作台静压导轨的工程设计计算具有理论指导意义。

(2) 结合CKS5235数控立式车床工作台静压导轨油 膜的速度场、压力场和温度场分析结果, 对静压导轨结构 进行优化, 优化后工作台的最高转速由 $63 \mathrm{r} / \mathrm{min}$ 提高到 $120 \mathrm{r} / \mathrm{min}$, 完成了重大专项的技术验收。 


\section{致谢}

本文为国家科技重大专项“高档数控机床与基础制造 装备”课题《超重型数控单柱移动立式铣车床》 (No.2011ZX04002-091) 的成果之一。

\section{参考文献}

[1] 刘赵炎, 金秋颖,张成印等.计入温-粘及压-粘关系式的油膜 工作性能数值分析[J]. 计算力学学报, 2011,28:69-73。

[2] 邵俊鹏,张艳芹,于晓东等.重型静压轴承扇形腔和圆形腔温 度场数值模与分析 [J]. 水动力学研究与进展 $\mathrm{A}$ 辑,2009,24(01):119-124。

[3] 王少力, 熊万里,孟曙光等. 恒流供油扇形静压推力轴承承载 力解析计算与实验研究 [J].机械强度 $2015,37(5)$ : 828-832。
[4] 何发诚,桂林.变频恒流静压轴承的研制,制造技术与机 床,2011,9:140-141。

[5] 邵俊鹏,张艳芹,李鹏程. 基于FLUENT的静压轴承椭圆腔和 扇形腔静止状态流场仿.2007,32(1):93-95。

[6] 张艳芹, 邵俊鹏, 韩桂华等. 大尺寸扇形静压推力轴承润 滑性能的数值分析.机床与液压.2009,37(1):69-71。

[7] 张艳芹, 邵俊鹏, 倪世钱. 大尺寸静压轴承温度场数值模 拟[J].中国机械工程，2008，19(5): 563-565。

[8] 赵明黄正东王书亭等. 重型数控立车工作台静压计算. 机 械工程学报.2009,45(9):120-125。

[9] 王巧玲.静压导轨的流体-结构耦合分析及动态性能优化设 计 [J].精密制造与自动化,2015,(1):24-30。

[10] 杨甫,余光怀,张力等. 回转工作台闭式静压导轨优化设计与 研究[J].制造技术与机床,2017,(7):71-74。 